\title{
HL7 Clinical Document Architecture to Share Cardiological Images and Structured Data in Next Generation Infrastructure
}

\author{
P Marcheschi, A Mazzarisi, S Dalmiani, A Benassi \\ Institute of Clinical Physiology, CNR, Pisa, Italia
}

\begin{abstract}
In medicine and in cardiology different standards are used for treatment of clinical and iconographic information. Among the most relevant there are HL7 for clinical data and DICOM for images and signals. The advent of Electronic Health Record Systems (EHR) and the request for data integration coming from different imaging modalities and diagnostic instrumentation, offer us a technological panorama difficult to manage. There is an increasing demand to select the most meaningful information in a simple and effective way, without the duty and the necessity to create from scratch new ways of data communication and sharing. Hence an agreement, in order to be able to feed a new multidisciplinary information databases, is necessary. To reach this goal, we used the emergent development of HL7 in the field of the structure of Clinical Document Architecture (CDA).
\end{abstract}

\section{Introduction}

In the field of specialistic medicine and particularly in cardiology it makes sense to do a minute structuring of the information that is acquired through the various interventistic procedures and from connected laboratories. The reason for this type of data collection is given by demand for improvement of better patient care quality that can become a reality in this way. The diffusion of standards in medical field is a recent success, but this trend is destined to grow, together with the diffusion of the XML language. The XML language has given a real turning point to the way of treating the information in all human knowledge fields. In medicine, from one side, allows the structuring of the data, from another side allows the elimination of proprietary formats that have tormented the existence of ICT software developers. From a developer point of view to embrace a new technology also means to learn new programming tools. But this difficulty in using XML can be overcome by the use of existing and innovative development tools already present in the opensource software panorama. For example the JAVA platform, permits directly to process
XML files, with al large number of tool, for example: Java API for XML Processing (JAXP) or Java Architecture for XML Binding (JAXB) [1,2].

"HL7", is a not-for-profit standards developing organization that provides standards "for the exchange, management and integration of data that supports clinical patient care and the management, delivery and evaluation of healthcare services".

CDA, the HL7 Clinical Document Architecture uses the eXtensible Markup Language (XML) with a semantic structure that allows a profitable use of well known multimedia formats joined with the data of clinical pertinence: instrumental examinations reports, chemical laboratories results, administrative data, etc. The overall view of a patient status is composed by a multitude of heterogeneous information, represented with the use of images, cine-loops and waveforms, textually with collected laboratory reports and related information. HL7 CDA Release 2 represents an important vector for future technological developments in medical-computer science. It offers manifold improvements on the forehead of the interoperability: It is human readable and independent from the database implementation or from the development environment, moreover it offers the availability in the same document of structured and unstructured data elements. The purpose of this work is to provide some guide-lines for the implementation and the treatment of the cardiological data using CDA architecture, through different examples of use implemented in our research environment, establishing the base for a real integration and reuse of data inside the cardiological patient care workflow.

\section{Methods}

CDA provides an exchange model for clinical documents, it offers a great freedom in structuring a document, so that it is possible to use it for discharge summaries, clinical notes, referral, progress notes, public health records or for internal processing documents, such as interventistic data recording protocols.

In Institute of Clinical Physiology CNR, we face every 
day the problem to collect data in the more simple and effective way, and every day physicians want new data to be added to our patient health record. CDA helps us to make possible to build an application from scratch reusing code already developed for other projects.

CDA offers an highly structured and at the same time highly modulable container. CDA structure is very simple, it consists of two main parts:

- The Header

- The Body.

The header contains service information, and necessary elements for the identification of the executed proce-dure. It permits the clinical document exchange and management. It contains the destination of the document, affiliation of the document, version control fields, to manage relationship with other documents. In Fig.1 is shown an example extracted from the header. The header contains also the authenticator informations needed for legal reason, and the author informations. The author of the document, could be a human being or, as often happens, a program that produces it, in algorithmic way. The body contains the clinical-related information that indeed we want to exchange. It can be classified in two main typologies: structured (StructuredBody) or not structured (NonXMLBody).

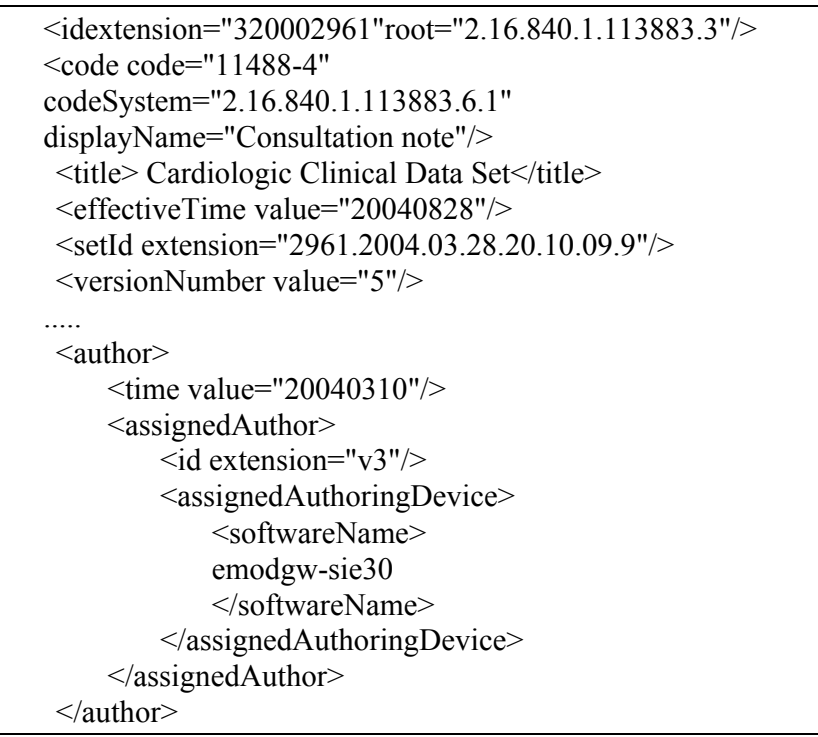

Figure 1. Presents an example extracted from the Header part of the CDA release 2 document.

CDA with unstructured body can be used in a great variety of circumstances: it is possible to include an object of any other type (i.e. Binary file such as an Acrobat document or DICOM file) as subject of clinical transferal. CDA with structured body allows a specific and com-plete exchange of clinical data structure.
By the definition of a CDA pattern, an agreement between sender and receivers, we are able to map desired clinical data in conventional mode. Looking into Fig. 2 and 3 clinical data has been organized as a collection of segments called components, each of ones is organized as a collection of entry that specify the single structured data.

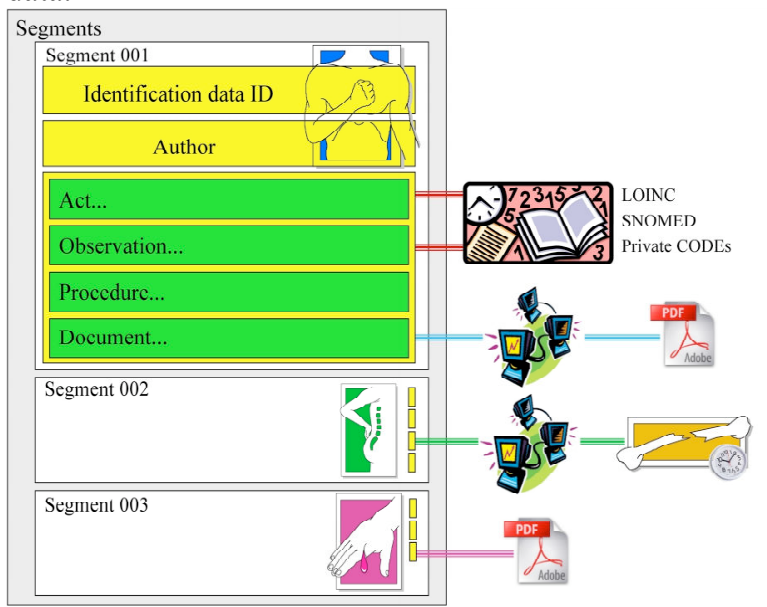

Figure 2. Structured Body Schema.

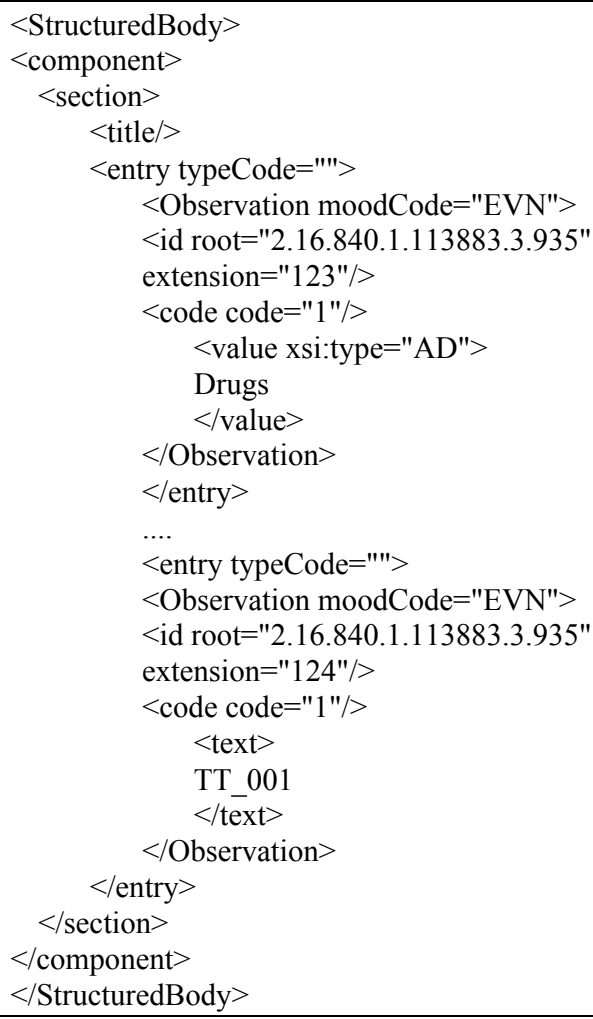

Figure 3. Extract from CDA XML body. 
Each component contains a section that is customizable with an Identification data ID, Author, and other tags that specify exactly the content. The most notable feature of a section is that it can contain 4 kind of external references (Act, Observation, Procedure, Document), infact there is the choice to include an inline object or to reference it with a web or telecom address. For every external link it is possible to specify one or more "useableTime", a period of time during which is possible to reach the shared resource. In this manner it is possible, as an example, to link an external DICOM file, present on an external repository that is active only for a certain period of time, in order, for example to schedule its retrieval. This possibility opens a vast range of options, infact it can use Retrieve Information for Display (RID) repositories to retrieve DICOM images or waveform data, or it can use a conventional HTTP/FTP server. The choice of body type is dictated by the demand of compatibility with other standards present in information and communication technology field, for instance ADOBE PDF or Microsoft word documents, but in our opinion the choice to prefer is the structured body with the purpose to fully exploit the possibilities offered by this mechanism of communication, and to respect the human readability guide-line.

However the need to include files of big dimensions, images choosen by medical doctors or waveforms in picture format, inside a clinical document is felt as a duty for the safety of the patient, but this can lead to a deceleration of the overall system responsiveness.

In the case, where is chosen to insert a link to an original image present on a remote file server, some essential characteristics of the image have to be known: Color depth, array dimension, number of frames, compression type. This in order to warn the end user and the query program about the necessary requirements to download the linked files, and in order to display correctly the downloaded images or cine-loops.

The usage of DICOM images is the solution to be preferred, in comparison to the use of jpeg or gif images, that don't have all the necessary information (i.e. pixel size, pixel depth, window/level settings, person name, age, sex etc..) for a correct medical evaluation. It is clear that the information present in the DICOM file has to coincide with that present into the header of the CDA, to avoid ambiguous information spreading.

One of the key feature of CDA and XML is the possibility to be verified with the help of an XML schema, this it is a powerful tool in the hands of a developer that wants to verify the validity of a clinical document. This type of validation is syntactic and not semantic, but it provides a valid tool to disclose wrong implementations of the standard.

In order to supply a confortable and ready tool for the developers community we have published a web service that offers this kind of CDA validation, accessible through the page of HL7 italian site, see Fig. 4: (http://www.ifc.cnr.it/hl7).

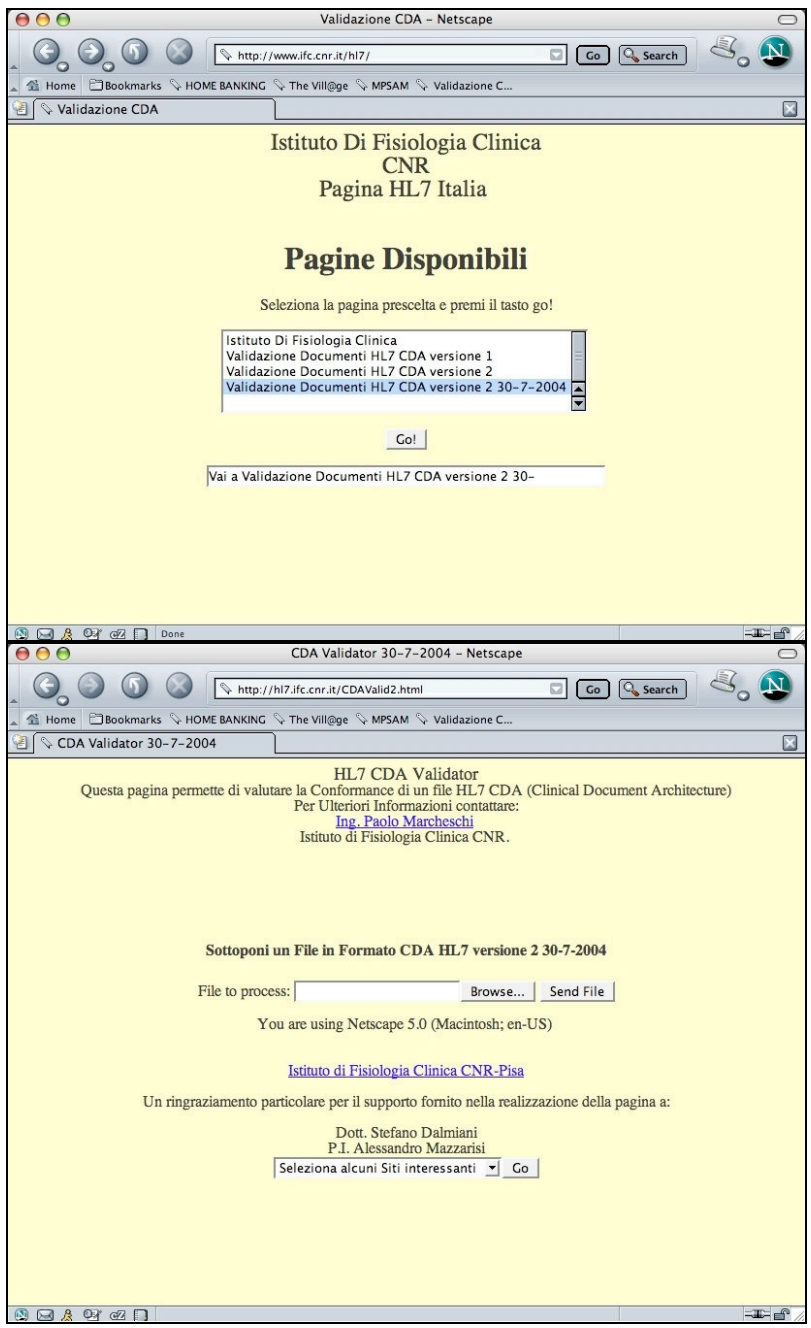

Figure 4. HL7 CDA Validation tools.

This web service can be invoked with the specialized page present on the site, or directly from a program trough a HTTP-POST method. The output of the web service is a self explanatory web page that shows the errors eventually present in the processed document.

Moreover CDA, is the element that lie at the base of the new IHE integration profile XDS: Cross enterprise Document Sharing [8].

This type of communication will allow to extend more the complete set of services provided by IHE initiative. $\mathrm{XDS}$ is a data integration system based upon the emerging ebxml $[9,10]$ technology, it uses CDA document as the election kind of document, and a system of ebxml registry/repositories to store and retrieve 
documents.

The creation of a CDA document represents the most complicated part of the development process, first, we have to reduce the fields tightly allowed by the verification scheme and to choose the necessary fields to map completely the selected application to develop.

To send CDA documents with structured body to an EHR system, for example, we had to define a CDA Pattern coming from an analysis of requested data definition in order to establish a set of clinical data to be exchanged.

Besides it is necessary to choose the more comfortable way to structure the data inside the CDA document. This because different ways exist for structuring the info, it is for instance possible to store the data coming from a homogeneous group inside elements of a chart, or it is possible to store them inside fields marked by an ID tag.

The choice of a method rather than another is not only dictated by a requirement of internal representation of the local database, but also from the need to respect the human readability of the created document. Human readability is a requisite that allows a greater intelligibility of the document and it avoids the creation of some incomprehensions among two parties. Besides it allows the application of the XML stylesheets [11,12], so that to represent automatically, for example, whole or partly the information in the document as an HTML or as a Adobe PDF page.

The application of a style-sheet is an automatic process, that can be performed in the greater part of actual internet browsers, like Mozilla Firefox or Internet Explorer.

\section{Conclusions}

The extensive use of HL7 CDA is desirable, not only in cardiology environment but also for all the other field present in medicine. This because it can easily developed and deployed. The ease of use descends from the great use of XML language for structuring documents; from the possibility to validate the document produced protect the developer from the improper use; from the ready available programming libraries to parse an XML document and as a consequence, an HL7 CDA document.

In the CNR Institute of Clinical Physiology, we have developed several number of application using webservices and HL7 CDA documents in order to structure the data.
We found that this approach can be useful to integrate legacy systems with the new technology generation applications so that to protect our old investiments. The use of web-services is dictated by the need of code reuseability and use of distributed information syatems. The adoption of new standards becomes gradually less difficult, compared to existing interchange formats, yelding to a faster technology advance process. The freedom to implement whatever we want is respected..

\section{References}

[1] Gosling J, Joy B, Steele G, Bracha G: The Java Language Specification Second Edition. Addison-Wesley; 2000.

[2] McLaughlin B. Java \& XML, 2nd Edition: Solutions to Real-World Problems. O'Reilly

[3] Public HL7-CDA Validation page: http://www.ifc.cnr.it/hl7 accessed september 2004

[4] Robert H. Dolin, MD, Liora Alschuler, Calvin Beebe, et al.: The HL7 Clinical Document Architecture American Medical Informatics Association J Am Med Inform Assoc. 2001 November; 8 (6): 552-569

[5] Frazier P, Rossi-Mori A, Dolin RH, Alschuler L, Huff SM. The creation of an ontology of clinical document names. In: Patel V, Rogers R. Haux R (eds). MedInfo 2001. Studies in Health Technology and Informatics, vol 84. Amsterdam, The Netherlands: IOS Press, 2001

[6] HL7 Reference Information Model (RIM) and the HL7 Version 3 Meta-Model for the Message Development Framework.http://www.hl7.org/library/datamodel/index.cfm. accessed september 2004

[7] A Mazzarisi, P Marraccini, P Marcheschi, et al.: Fully Integrated Hemodynamic Interventional Laboratory Informative System Computers in Cardiology 2003;30:403-406

[8] Parisot C. et al.: IHE IHE IT Infrastructure Technical Framework Supplement 2004-2005 Cross-Enterprise Document Sharing (XDS).

[9] ebXML Technical Architecture Specification v1.0.4 http://www.ebxml.org/specs/ebTA.doc accessed september 2004

[10] http://ebxmlrr.sourceforge.net/presentations/freebXMLReg istryBrochure.pdf. accessed september 2004.

[11] Harold ER:Processing XML with Java:A Guide to SAX, DOM, JDOM, JAXP, and TrAx Addison-Wesley 2001.

[12] Rockwell W.: XML, XSLT, Java, and JSP: A Case Study in Developing a Web Application, New Riders Press; Bk\&CD-Rom edition, 2001.

Address for correspondence

Paolo Marcheschi

Via G.Moruzzi 1, 56100 Pisa, Italia

paolo@ifc.cnr.it 